\title{
Obligatorische Qualitätskontrolle 2004
}

\author{
Version 2004
}

QUALAB Schweizerische Kommission für Qualitätssicherung im medizinischen Labor

\section{Bestimmungen}

Auszüge aus dem Konzept QUALAB, nicht umfassend.

Konzept QUALAB, Art. 4.2.1.1 Abs. 1

«Jedes Laboratorium nimmt für seine Analysen an mindestens 4 externen Ringversuchen pro Jahr teil $[\ldots] . »$

Konzept QUALAB, Art. 4.2.1.1 Abs. 7

«Die Liste Obligatorische Qualitätskontrolle hält diejenigen Analysen der Analysenliste fest, für die eine externe Qualitätskontrolle obligatorisch ist $[\ldots]$.»

\section{Konzept QUALAB, Art. 4.2.3.2}

«Medizinische Laboratorien, die innert 2 Jahren nicht an den vorgeschriebenen 8 Ringversuchen teilgenommen haben, werden von allen Versicherern für $1 \mathrm{Jahr}$, im Wiederholungsfalle dauernd von der Bezahlung ausgeschlossen.»

\section{Konzept QUALAB, Art. 4.2.3.3 Abs. 1}

«Liegen die Messgenauigkeiten pro Analyse ausserhalb der von der QUALAB festgelegten Beurteilungskriterien (siehe Liste der obligatorischen Qualitätskontrolle), so wird eine Expertise auf Kosten des fehlbaren medizinischen Laboratoriums angeordnet. Die Expertise und Beratung umfasst die gesamte Qualitätssicherung gemäss QUALAB/KBMAL.»

Konzept QUALAB, Art. 4.2.3.3 Abs. 2

«Das medizinische Laboratorium, dessen externe Qualitätskontrollresultate ungenügend sind, ist in jedem Fall gehalten, Beratung und Hilfe zu suchen.»

\section{Liste der anerkannten Qualitäts- kontrollzentren (Stand 1. Januar 2004)}

\section{Schweiz}

a CSCQ Schweizerisches Zentrum für Qualitätskontrolle

CSCQ, Chemin du Petit-Bel-Air 2, 1225 ChêneBourg, Tel. 0223055231 (deutsch), 0223055230 (französisch), 0223055232 (italienisch), Fax 02230552 38, E-Mail: cscq@hcuge.ch, Internet: www.cscq.ch

b MQ Verein für medizinische Qualitätskontrolle MQ, c/o Institut für klinische Chemie, Universitätsspital, 8091 Zürich, Tel. 0125534 11, Fax 0126112 83, E-Mail: mq@unizh.ch, Internet: www.mqzh.ch

C BSD SRK Blutspendedienst SRK Bern AG

BSD SRK Bern AG, Murtenstrasse 133, Postfach 5512, 3001 Bern, Tel. 03138423 23, Fax 03138423 24, E-Mail: christoph.niederhauser @bsd-be.ch

\section{Ausland}

d EMQN European Molecular Genetics Network EMQN, c/o Regional Molecular Genetics Laboratory, St Mary's Hospital, Hathersage Road, UKManchester M13 0JH, Tel. 0044161276 6741, Fax 0044161276 6606, E-Mail: office@emqn.org, Internet http://www.emqn.org/emqn.htm

\section{e BVMG Deutscher Berufsverband}

für Medizinische Genetik

BVMG, Goethestrasse 29, D-80336 München, Tel. 0049895502 7855, Fax 0049895502 7856, E-Mail: bv@medgenetik.de, Internet http:// bvmedgen.de/

f UK NEQAS United Kingdom National External Quality Assessment Schemes (nur für Genetik) UK NEQAS Office, PO Box 401, UK-Sheffield S5 7YZ, Tel. 0044114261 1689, Fax 0044114261 1049, E-Mail: office@ukneqas.org.uk, Internet http://www.ukneqas.org.uk/ 


\section{Listen der Analysen, die per 1. Januar 2004 der obligatorischen externen Qualitätskontrolle unterworfen sind}

\subsection{Chemie, Hämatologie, Immunologie,} Mikrobiologie

$\mathrm{M}=$ Modifikationen gegenüber 2003: $\mathrm{N}=$ neu auf der Liste; $C=$ Änderung Kriterium; $S=$ Streichung; $\mathrm{Q}=$ Änderung anerkannte QKZ.
Die aufgeführten Parameter sind auch dann der obligatorischen externen Qualitätskontrolle unterworfen, wenn sie im Rahmen eines Analysenblocks unter einer anderen Nummer abgerechnet werden.

(diese Liste geht auf der nächsten Seite weiter)

\begin{tabular}{|c|c|c|c|c|c|}
\hline M & Pos.-Nr. & Parameter & $\begin{array}{l}\text { Beurteilungs- } \\
\text { kriterien }\end{array}$ & $\begin{array}{l}\text { Erfüllungs- } \\
\text { kriterien }\end{array}$ & Anerkannte QKZ \\
\hline & 8000.00 & Blutgruppen & richtig & $11 / 12$ & $\mathrm{C}$ \\
\hline & 8006.00 & Alanin-Aminotransferase (ALAT) & $\pm 25 \%$ & $75 \%$ & $a, b$ \\
\hline & 8007.00 & Albumin, chemisch & $\pm 15 \%$ & $75 \%$ & $a, b$ \\
\hline & 8012.00 & Alkalische Phosphatase & $\pm 25 \%$ & $75 \%$ & $a, b$ \\
\hline & 8036.00 & Amphetamine, ql & richtig & $75 \%$ & $a, b$ \\
\hline & 8037.00 & Amylase & $\pm 40 \%$ & $75 \%$ & $a, b$ \\
\hline & 8058.00 & Aspartat-Aminotransferase (ASAT) & $\pm 25 \%$ & $75 \%$ & $a, b$ \\
\hline & 8116.00 & Barbiturate, ql & richtig & $75 \%$ & $a, b$ \\
\hline & 8119.00 & Benzodiazepine, ql & richtig & $75 \%$ & $a, b$ \\
\hline & 8126.00 & Bilirubin total & $\pm 20 \%$ & $75 \%$ & $a, b$ \\
\hline & 8129.00 & Blutgase $\left(\mathrm{pO}_{2}, \mathrm{pCO}_{2}, \mathrm{pH}\right)$ & $\pm 20 \%$ & $75 \%$ & $a, b$ \\
\hline & 8137.00 & C-reaktives Protein, qn & $\pm 20 \%$ & $75 \%$ & $a, b$ \\
\hline & 8149.00 & Calcium, total & $\pm 12 \%$ & $75 \%$ & $a, b$ \\
\hline & 8156.00 & Chlorid & $\pm 10 \%$ & $75 \%$ & $a, b$ \\
\hline & 8158.00 & Cholesterin total & $\pm 10 \%$ & $75 \%$ & $a, b$ \\
\hline & 8169.00 & Cocain, ql & richtig & $75 \%$ & $a, b$ \\
\hline & 8179.00 & D-Dimere, ql & richtig & $75 \%$ & $a, b$ \\
\hline & 8179.01 & D-Dimere, qn & $\pm 30 \%$ & $75 \%$ & $a, b$ \\
\hline & 8184.00 & Cannabis, ql & richtig & $75 \%$ & $a, b$ \\
\hline & 8192.00 & Eisen & $\pm 20 \%$ & $75 \%$ & $a, b$ \\
\hline & 8199.00 & Erythrozyten Alloantikörper, Spezifizierung & richtig & $100 \%$ & c \\
\hline & 8200.00 & Erythrozyten Alloantikörper, Suchtest & richtig & $100 \%$ & c \\
\hline & 8210.00 & Erythrozytenzählung & $\pm 25 \%$ & $75 \%$ & $a, b$ \\
\hline \multirow[t]{2}{*}{$\mathrm{N}$} & 8225.00 & Ferritin & $\pm 25 \%$ & $75 \%$ & $a, b$ \\
\hline & 8229.00 & Fibrinogen, nach Clauss & $\pm 15 \%$ & $75 \%$ & $a, b$ \\
\hline \multirow[t]{9}{*}{$\mathrm{N}$} & 8239.00 & Folat & $\pm 20 \%$ & $75 \%$ & $a, b$ \\
\hline & 8249.00 & Gamma-Glutamyltranspeptidase (@-GT) & $\pm 25 \%$ & $75 \%$ & $a, b$ \\
\hline & 8259.00 & Glucose & $\pm 10 \%$ & $75 \%$ & $a, b$ \\
\hline & 8265.00 & Glykiertes Hämoglobin (HbA1c) & $\pm 15 \%$ & $75 \%$ & $a, b$ \\
\hline & 8273.00 & Hämatokrit & $\pm 12 \%$ & $75 \%$ & $a, b$ \\
\hline & 8275.00 & Hämoglobin & $\pm 12 \%$ & $75 \%$ & $a, b$ \\
\hline & 8284.00 & Harnstoff & $\pm 20 \%$ & $75 \%$ & $a, b$ \\
\hline & 8288.00 & HDL-Cholesterin, ohne separate Fällung & $\pm 30 \%$ & $75 \%$ & $a, b$ \\
\hline & 8313.00 & Immunglobuline IgA (Serum) & $\pm 25 \%$ & $75 \%$ & $a, b$ \\
\hline \multirow[t]{2}{*}{$\mathrm{N}$} & 8317.00 & Immunglobulin IgE total, qn & $\pm 20 \%$ & $75 \%$ & $a, b$ \\
\hline & 8317.01 & Immunglobulin IgE multispezifisch & richtig & $75 \%$ & $a, b$ \\
\hline $\mathrm{N}$ & 8317.02 & Spezifisches IgE - Erdnuss & \pm 1 Klasse & $75 \%$ & a \\
\hline $\mathrm{N}$ & 8317.02 & Spezifisches lgE - Birke & \pm 1 Klasse & $75 \%$ & a \\
\hline N & 8317.02 & Spezifisches IgE - Katzenepithel & \pm 1 Klasse & $75 \%$ & a \\
\hline
\end{tabular}




\begin{tabular}{|c|c|c|c|c|c|}
\hline $\mathrm{N}$ & 8317.03 & Spezifisches IgE - Erdnuss qn & $\pm 20 \%$ & $75 \%$ & a \\
\hline N & 8317.03 & Spezifisches lgE - Birke qn & $\pm 20 \%$ & $75 \%$ & a \\
\hline \multirow[t]{17}{*}{$\mathrm{N}$} & 8317.03 & Spezifisches IgE - Katzenepithel qn & $\pm 20 \%$ & $75 \%$ & a \\
\hline & 8318.00 & Immunglobuline IgG (Serum) & $\pm 25 \%$ & $75 \%$ & $a, b$ \\
\hline & 8321.00 & Immunglobuline IgM (Serum) & $\pm 25 \%$ & $75 \%$ & $a, b$ \\
\hline & 8343.00 & Kalium & $\pm 10 \%$ & $75 \%$ & $a, b$ \\
\hline & 8384.00 & Kreatin-Kinase (CK), total & $\pm 40 \%$ & $75 \%$ & $a, b$ \\
\hline & 8387.00 & Kreatinin & $\pm 20 \%$ & $75 \%$ & $a, b$ \\
\hline & 8394.00 & Laktat-Dehydrogenase (LDH) & $\pm 25 \%$ & $75 \%$ & $a, b$ \\
\hline & 8403.00 & Differenzialblutbild & * & $75 \%$ & $a, b$ \\
\hline & 8406.00 & Leukozytenzählung & $\pm 25 \%$ & $75 \%$ & $a, b$ \\
\hline & 8428.00 & Magnesium & $\pm 20 \%$ & $75 \%$ & $a, b$ \\
\hline & 8433.00 & Methadon, ql & richtig & $75 \%$ & $a, b$ \\
\hline & 8437.00 & Myoglobin & $\pm 30 \%$ & $75 \%$ & $a, b$ \\
\hline & 8438.00 & Natrium & $\pm 10 \%$ & $75 \%$ & $a, b$ \\
\hline & 8444.00 & Opiate, ql & richtig & $75 \%$ & $a, b$ \\
\hline & 8450.00 & Pankreas-spezifische Amylase & $\pm 25 \%$ & $75 \%$ & $a, b$ \\
\hline & 8455.10 & $\begin{array}{l}\text { aktivierte partielle } \\
\text { Thromboplastinzeit (aPTT) }\end{array}$ & $\pm 25 \%$ & $75 \%$ & $a, b$ \\
\hline & 8462.00 & Phosphat & $\pm 15 \%$ & $75 \%$ & $a, b$ \\
\hline $\mathrm{N}$ & 8485.00 & Prostata-spezifisches Antigen (PSA) & $\pm 25 \%$ & $75 \%$ & $a, b$ \\
\hline \multirow[t]{4}{*}{ N } & 8485.01 & Prostata-spezifisches Antigen (PSA), freies & $\pm 25 \%$ & $75 \%$ & $a, b$ \\
\hline & 8492.00 & Protein total & $\pm 15 \%$ & $75 \%$ & $a, b$ \\
\hline & 8548.00 & Quick & $\pm 15 \%$ & $75 \%$ & $a, b$ \\
\hline & 8560.00 & Thrombozytenzählung & $\pm 25 \%$ & $75 \%$ & $a, b$ \\
\hline \multirow[t]{3}{*}{ N } & 8562.00 & Thyroxin, freies (FT4) & $\pm 20 \%$ & $75 \%$ & $a, b$ \\
\hline & 8572.00 & Triglyceride & $\pm 20 \%$ & $75 \%$ & $a, b$ \\
\hline & 8574.10 & Troponin (T oder I), mittels ELISA & $\pm 30 \%$ & $75 \%$ & $a, b$ \\
\hline N & 8576.00 & Thyreotropin (TSH), basal & $\pm 20 \%$ & $75 \%$ & $a, b$ \\
\hline \multirow[t]{3}{*}{ N } & 8577.00 & Thyreotropin (TSH), stimuliert & $\pm 20 \%$ & $75 \%$ & $a, b$ \\
\hline & 8578.00 & Harnsäure & $\pm 20 \%$ & $75 \%$ & $a, b$ \\
\hline & 8587.00 & $\begin{array}{l}\text { Verträglichkeitstest, } \\
\text { ind. Anti-Humanglobulintest }\end{array}$ & richtig & $100 \%$ & c \\
\hline \multirow[t]{6}{*}{$\mathrm{N}$} & 8590.00 & Vitamin B12 & $\pm 20 \%$ & $75 \%$ & $a, b$ \\
\hline & 9108.40 & Hepatitis-B-Virus-HBs-Antigennachweis, ql & keine falsch neg. & $100 \%$ & $a, c$ \\
\hline & 9109.01 & Hepatitis-C-Virus-Antikörper IG oder IgG, qI & keine falsch neg. & $100 \%$ & $a, c$ \\
\hline & 9116.01 & HIV-1+2-Antikörper (EIA), ql & keine falsch neg. & $100 \%$ & $a, c$ \\
\hline & 9116.40 & $\begin{array}{l}\text { HIV-1+2-Antikörper } \\
\text { (Screening) Schnelltest, ql }\end{array}$ & keine falsch neg. & $100 \%$ & $a, b$ \\
\hline & 9309.00 & Urin, Eintauchobjektträger & richtig & $75 \%$ & $a, b$ \\
\hline
\end{tabular}




\subsection{Genetik}

Aus technischen Gründen wird für diese Analysen nur ein Ringversuch pro Jahr angeboten. Dabei müssen aber mehrere Proben untersucht werden.

Alle Laboratorien, welche die untenstehenden Analysen durchführen, müssen jedes Jahr sofort nach Erhalt, aber spätestens per Ende Jahr, eine Kopie der Auswertungen bzw. des Zertifikats an die QUALAB schicken.

$M=$ Modifikationen gegenüber 2003: $N=$ neu auf der Liste; $C=$ Änderung Kriterium; $Q=$ Änderung anerkannte QKZ.

\begin{tabular}{|c|c|c|c|c|c|}
\hline \multirow[t]{10}{*}{ M } & Pos.-Nr. & Parameter & $\begin{array}{l}\text { Beurteilungs- } \\
\text { kriterien }\end{array}$ & $\begin{array}{l}\text { Erfüllungs- } \\
\text { kriterien }\end{array}$ & Anerkannte QKZ \\
\hline & 8801.00 & Konstitutioneller Karyotyp & richtig & $1 / 1$ & $e, f$ \\
\hline & 8802.00 & $\begin{array}{l}\text { Chromosomenunters., } \\
\text { maligne Hämopathien }\end{array}$ & richtig & $1 / 1$ & $f$ \\
\hline & 8805.00 & FISH-Metaphase & richtig & $1 / 1$ & $e, f 7$ \\
\hline & 8806.00 & FISH-Interphase & richtig & $1 / 1$ & $e, f$ \\
\hline & 8810.01 & Angelman / Prader Willi & richtig & $1 / 1$ & $d, e$ \\
\hline & 8810.05 & Y-Chromosom-Mikrodeletion & richtig & $1 / 1$ & $d$ \\
\hline & 8810.07 & Cystische Fibrose & richtig & $1 / 1$ & d, e \\
\hline & 8810.08 & Fragiles X-Syndrom & richtig & $1 / 1$ & d, e \\
\hline & 8810.11 & Hämochromatose & richtig & $1 / 1$ & $a, b, e$ \\
\hline \multirow[t]{5}{*}{ N } & 8810.13 & Hämostasestörungen & richtig & $1 / 1$ & $a, b$ \\
\hline & 8810.19 & $\begin{array}{l}\text { Brustkrebs, hereditäre non-polypöse } \\
\text { Colonkarzinome / Retinoblastom }\end{array}$ & richtig & $1 / 1$ & $d, e$ \\
\hline & 8810.23 & Duchenne Muskeldystrophie & richtig & $1 / 1$ & d, e \\
\hline & 8810.27 & $\begin{array}{l}\text { Myotone Dystrophie / Friedreich'sche Ataxie / } \\
\text { Huntington Disease / Spinocerebelläre } \\
\text { Ataxien }\end{array}$ & richtig & $1 / 1$ & e \\
\hline & 8810.32 & Charcot-Marie-Tooth & richtig & $1 / 1$ & $d, e$ \\
\hline
\end{tabular}

\section{Beurteilung der obligatorischen Qualitätskontrolle}

Bei der Beurteilung der Resultate der externen Qualitätskontrolle müssen folgende Punkte berücksichtigt werden:

- In der Labormedizin sind sowohl die Analysemethoden wie auch die verwendeten Analysensysteme sehr vielfältig. Dies kann zu einer Heterogenität der Qualitätskontrollresultate führen. Einheitliche Qualitätskriterien bzw. Sollwerte sind deshalb nicht immer festlegbar.

- Die Qualität der Ringversuchsresultate wird beeinflusst durch die Qualität des Ringversuchsmaterials. Es ist aus technischen Gründen nicht immer möglich, für jedes Analysensystem ein optimal geeignetes Kontrollmaterial zur Verfügung zu stellen. Die Qualitätskontrollzentren müssen dieses Element bei der Beurteilung der Resultate mit einbeziehen können.
- Bei der Beurteilung der Qualität der Resultate der Ringversuche müssen aus den oben erwähnten Gründen mehrere Spezialfälle berücksichtigt werden. Diese im voraus einzeln zu kennen ist nicht möglich. Den Qualitätskontrollzentren wird deshalb versuchsweise für ein Jahr ein gewisser Ermessensspielraum eingeräumt, damit die Ringversuche rasch und unbürokratisch durchgeführt und ausgewertet werden können.

Dieser einjährige Ermessensspielraum gilt auch für neu einzuführende Parameter. Die Qualitätskontrollzentren berichten Ende Jahr über spezielle Vorkommnisse, bei denen dieser Spielraum beansprucht wurde. 


\subsection{Parameter mit oder ohne obligatorische Qualitätskontrolle}

\subsubsection{Parameter mit obligatorischer Qualitäts-} kontrolle (gemäss Kapitel 3 des vorliegenden Dokuments):

Die jährliche Teilnahme an mindestens 4 Ringversuchen (Abgabe von 4 Resultaten pro Analyse) ist obligatorisch. Verlangt die QUALAB in Ausnahmefällen oder für gewisse Spezialanalysen nur zwei Ringversuche, so ist die Teilnahme an diesen 2 Ringversuchen erforderlich.

Die Qualität der Analytik wird entsprechend den durch die QUALAB festgesetzten Kriterien beurteilt.

\subsubsection{Parameter ohne obligatorische externe} Qualitätskontrolle (nicht auf der Liste von Kapitel 3): Es existieren keine durch die QUALAB aufgestellten Richtlinien (Qualitätskriterien).

\subsection{Beurteilungskriterien/Zertifikat}

\subsubsection{Beurteilungskriterien, Erfüllungskriterien}

Beurteilt wird die Qualität der Analytik jedes Parameters im Jahresverlauf. In der Regel sind die Anforderungen dann erfüllt, wenn pro Parameter $75 \%$ der Resultate der Ringversuche eines Jahres den Anforderungen gemäss QUALAB entsprechen. Bestimmte Parameter können andere Anforderungen haben (siehe Kolonne «Erfüllungskriterien», Tabelle Kapitel 3).

\subsubsection{Zertifikat}

Das Zertifikat enthält eine Liste der vom Laboratorium gemeldeten und $\mathrm{zu}$ kontrollierenden Parameter (mit Positionsnummer der AL), die gesamte Anzahl der Ringversuche pro Parameter und die Anzahl der konformen Resultate. Das Zertifikat muss eine eindeutige Identifizierung, den Namen und die Adresse des Laboratoriums, seine ZSR-Nummer (ehemals Konkordatsnum- mer), die Kontrollperiode, die Identifizierung des ausstellenden QKZ sowie den Namen und Titel der Person, die das Zertifikat validiert, aufweisen.

\subsubsection{Neuanmeldungen / Ummeldungen}

Falls ein Labor eine neue Analyse einführt oder im Verlauf des Jahres neu mit der Qualitätskontrolle beginnt, muss das Zertifikat die Anzahl der zu liefernden Resultate pro rata temporis angeben.

Diese Teilnehmer sind von der Pflicht zur Untersuchung der nicht konformen Resultate und zum Protokollieren der getroffenen Massnahmen nicht befreit.

\subsection{Pflichten des Labors}

\subsubsection{Teilnahme an den Ringversuchen}

Wenn ein Labor Analysen gemäss Kapitel 4.1.1 durchführt, muss es sich für die erforderliche Zahl Ringversuche bei einem von der QUALAB anerkannten Qualitätskontrollzentrum anmelden. Für alle Parameter der Grundversorgung (siehe Eidgenössische Analysenliste) werden nur schweizerische Qualitätskontrollzentren anerkannt. Für alle anderen Parameter können auf Antrag der Fachgesellschaften auch ausländische Qualitätskontrollzentren anerkannt werden.

\subsubsection{Vorgehen bei Nichterfüllen der Beurteilungs-} kriterien

Das Labor ist verpflichtet, den Ursachen ungenügender Resultate auf den Grund zu gehen und die entsprechende Dokumentation aufzubewahren (mind. 5 Jahre).

Sind die Beurteilungskriterien und die Erfüllungskriterien für einen Parameter nicht erfüllt, muss das Labor zur Besprechung der Probleme mit dem Qualitätskontrollzentrum Kontakt aufnehmen, um den Ursprung des Problems zu suchen. 